\title{
Rôle des intermédiaires dans l'approvisionnement de l'industrie de transformation halieutique en région du Centre sud du Vietnam
}

\author{
Vinh Do Thi Thanh \\ Université de NhaTrang \\ 02, rue Nguyen Dinh Chieu \\ Nha Trang \\ Vietnam \\ <vinh.dothithanh@univ-nc.nc>
}

\begin{abstract}
Résumé
La transformation des produits halieutiques prend une place de plus en plus importante dans l'économie vietnamienne. L'étude de l'organisation de cette filière dans la région du Centre sud fait apparaître le rôle essentiel des intermédiaires qui achètent la production des pêcheurs et des aquaculteurs et les revendent aux entreprises de transformation. Leur influence ne se limite pas seulement à ce domaine, car ils financent en amont les pêcheurs et interviennent également dans la fixation des prix de vente de la matière première aux unités de transformation. Leur position prééminente se réalise malheureusement au détriment des intérêts des pêcheurs, dont le fonctionnement reste relativement artisanal, et influe sur le fonctionnement de l'entreprise de transformation halieutique. Après avoir examiné les contraintes spécifiques à cette situation, il convenait de formuler un certain nombre de propositions, tant au bénéfice des entreprises qu'à l'attention des décideurs, afin de rendre plus fonctionnel ce secteur d'activité. Généralisées, elles pourraient permettre au Vietnam - qui a entrepris, sur le plan économique, une transition maîtrisée vers l'économie de marché - de mener à bien une réforme profonde.
\end{abstract}

Mots clés : intermédiaire ; marché ; offre ; ressource halieutique ; transformation ; Vietnam.

Thèmes : pêche et aquaculture ; transformation, commercialisation.

\section{Abstract \\ Role of middlemen in the supply of the processing industry in the Central South region of Vietnam}

The processing industry is playing an increasingly important role in Vietnam's overall economy. A study of the restructuring of this sector across the Coastal Central South has demonstrated the dominant role that middlemen are taking in connecting the supply, represented by fishermen and farmers, to demand, represented by processing enterprises. Still, middlemen have gone further in establishing their influences over both, via assisting small producers in inputs for production while intervening in the price fixation traditionally practiced by processing enterprises. Indeed, their position of leverage works to the detriment of small-scale producers and processing enterprises. After examining specific constraints that have hampered potential development in the field, several recommendations are therefore proposed in favour of regulations and policy-marking processes at both business and industry levels. Once the tendency of extending this model is visible, it is hoped that suggested measures can encourage Vietnam, which has thus far passively witnessed the mainstream of transition from the centrally planned to market-oriented economy, to successfully introduce far-reaching reforms.

Key words: fishery resources; market; middle man; supply; transformation; Vietnam. Subjects: fishing and aquaculture; processing, marketing.

Tirés à part : $V$. Do Thi Thanh 
$\mathrm{D}$ ans un système performant d'approvisionnement de l'entreprise, les intermédiaires jouent souvent un rôle essentiel de fournisseurs. Le secteur de la transformation des produits halieutiques en fournit un bon exemple, que ce soit à travers les expériences des pays asiatiques ou celles d'autres pays en développement. C'est ainsi qu'au Nigeria, les intermédiaires peuvent être classés en trois groupes : les grossistes, les semigrossistes et les détaillants (Mafimisebi et Okunmadewa, 2006). Leurs activités sur le marché des produits aquatiques consistent à : regrouper, acheter, stocker, transporter, standardiser, faire de la publicité, approvisionner et vendre les produits. Au Bangladesh, aux Philippines, en Inde et en Thailande, le commerce des poissons est contrôlé par le secteur privé. Il existe quatre catégories d'intermédiaires qui interviennent entre les producteurs et les consommateurs finaux : les courtiers, les grossistes, les commerçants en gros et en détail, les détaillants (Dey et al., 2000a, 2002). C'est aussi le cas des entreprises de transformation halieutique du Vietnam, encore qu'il ne s'agisse pas vraiment d'un mécanisme identique. Nous nous proposons, dans le cadre de cette étude, d'examiner la situation telle qu'elle se présente dans la région du Centre sud qui présente de grandes similitudes avec la partie septentrionale du pays.

Dans la plupart des entreprises de la région du Centre sud du Vietnam, on ne choisit pas le canal d'approvisionnement direct entre le producteur de matière première et l'entreprise. Les fournisseurs intermédiaires y sont de plus en plus nombreux, et s'occupent des activités d'achat, de transport et de manutention de produits aquatiques destinés à l'entreprise. Certes, ils répondent mieux aux besoins de l'industrie de transformation halieutique, mais, en même temps, les contraintes induites par leurs activités "achat-vente" pèsent sur les producteurs et, parce qu'elles désorganisent parfois le système d'approvisionnement, le rendent difficile à gérer. Le problème de la régulation du système se pose donc en priorité dans la stratégie de développement de l'industrie de transformation halieutique dans cette région.

\section{Évolution historique}

Les intermédiaires, c'est-à-dire ceux qui prêtent de l'argent aux pêcheurs et collectent tout le produit de leurs captures, sont apparus en même temps que les activités de pêche dans la région du Centre sud du Vietnam.

Avant 1980, il s'agissait de petits collecteurs de produits aquatiques en vue de la consommation finale sur le marché local. À cette époque, l'exportation n'était pas encore développée, et le nombre des entreprises de transformation halieutique était limité. Le gouvernement gérait rigoureusement - par la planification et la subvention - l'approvisionnement et la distribution des entreprises.

À partir de l'année 1986, grâce à la rénovation des politiques économiques, le Vietnam s'est engagé dans une phase de transition vers l'économie du marché. Les activités d'exportation furent encouragées, stimulant ainsi la production des matières premières. Le taux d'augmentation annuel du nombre des entreprises dans les régions atteignit 17,6\% durant la période 1996-1999 (Thai Thanh, 2005). Cela entraîna évidemment l'accroissement du nombre des intermédiaires, ceux-ci jouant le rôle d'agents d'achat de l'entreprise. Durant cette période, les producteurs n'avaient pas encore intégré les données du marché, surtout en matière de prix d'achat par l'entreprise. Ils se trouvaient donc contraints de vendre à bon marché aux intermédiaires.

Avec les premières années du Xxi ${ }^{\mathrm{e}}$ siècle, le développement des moyens de communication permit aux pêcheurs d'obtenir facilement des informations sur le prix du marché pour les matières premières. Néanmoins, en raison de l'inefficacité de leurs activités d'achat, la plupart des entreprises durent concéder ces activités aux intermédiaires, ne jouant que le rôle de contrôleur de la qualité des matières premières. Actuellement, les activités des intermédiaires se stabilisent et se spécialisent.

\section{Perspectives de développement}

La région du Centre sud du Vietnam s'étend sur sept provinces et villes côtières, de la ville Da Nang jusqu'à la province de Binh Thuan. C'est la région où les activités de pêche se développent le plus : pêche côtière au chalut, à la senne, à la palangre; pêche hauturière. (tableau 1, tableau 2).

C'est aussi la région qui concentre la plupart des entreprises de transformation halieutique du Vietnam. Un des principaux facteurs de cet accroissement est la participation fonctionnelle des intermédiaires dans le système d'approvisionnement de ces entreprises.

Dans le système de distribution des produits pêchés, les intermédiaires jouent un rôle très important. Grâce au financement $\mathrm{du}$ projet ALMRV (Assessment of the Living Marine Resources in Vietnam) en 2005, les enquêtes qui ont été effectuées dans quelques provinces du Vietnam ont livré des statistiques impressionnantes: environ $90 \%$ des acheteurs du poisson débarqué sont des intermédiaires. Ils mâ̂trisent le marché au travers de l'achat des prises des pêcheurs et de la production des aquaculteurs, et de leur vente aux entreprises ou sur le marché local de la consommation intérieure.

La finalité de ce projet de recherche est d'étudier l'activité d'approvisionnement des intermédiaires en produits aquatiques destinés à la transformation halieutique, avec, en toile de fond, l'objectif de rendre celle-ci plus efficace.

\section{Méthodologie de recherche}

Au cours de cette recherche, nous avons utilisé la méthodologie quantitative fondée sur les statistiques disponibles ${ }^{1}$, afin de déterminer la contribution des intermédiaires dans la chaîne d'approvisionnement en produits aquatiques des entreprises. Par ailleurs, nous avons suivi la méthode d'analyse qualitative s'appuyant sur des enquêtes et entretiens menés auprès des acteurs de l'industrie de transformation sur le terrain, pour déterminer l'état des opérations d'achat et de fixation des prix entre eux. Une analyse synthétique tentera, en conclusion, de proposer des moyens d'amélioration de l'interaction entre les intermédiaires et les autres acteurs qui interviennent dans la chaîne d'approvisionnement de la région. Dans un premier temps, notre équipe de recherche a procédé à la visite de neuf grandes entreprises réparties dans les diverses régions maritimes de la région. Le choix de ces entreprises a fait l'objet

\footnotetext{
${ }^{1}$ Les statistiques des provinces de la région sont extraites de l'annuaire statistique du Vietnam. http://www.gso.gov.vn/default.aspx? tabid $=390 \&$ idmid $=3 \& \mid$ tem $\mid D=4760$
} 
Tableau 1. Nombre de navires de pêche hauturière dans la région du Centre sud du Vietnam

Table 1. Number of off-shore boats in the south-central region.

\begin{tabular}{|c|c|c|c|c|c|c|c|}
\hline & 2000 & 2001 & 2002 & 2003 & 2004 & 2005 & 2006 \\
\hline Du pays entier & 9766 & 14326 & 15988 & 17303 & 20071 & 20537 & 20807 \\
\hline Région du Centre sud & 5164 & 6837 & 7780 & 8258 & 9764 & 9662 & 9814 \\
\hline Ville de DaNang & 46 & 61 & 74 & 118 & 225 & 276 & 184 \\
\hline Province de QuangNam & 462 & 478 & 490 & 520 & 538 & 540 & 543 \\
\hline Province de QuangNgai & 540 & 1320 & 1452 & 1608 & 2393 & 1897 & 1993 \\
\hline Province de BinhDinh & 2795 & 2976 & 3431 & 3568 & 3656 & 3784 & 3903 \\
\hline Province de PhuYen & 82 & 375 & 402 & 420 & 650 & 755 & 911 \\
\hline Province de KhanhHoa & 415 & 430 & 430 & 458 & 609 & 665 & 620 \\
\hline Province de NinhThuan & 182 & 187 & 314 & 338 & 650 & 1055 & 1071 \\
\hline Province de BinhThuan & 642 & 1010 & 1187 & 1228 & 1043 & 690 & 589 \\
\hline
\end{tabular}

Unité : navire. Source : statistiques extraites de l'annuaire statistique par province.

Tableau 2. Statistiques de l'aquaculture dans la région du Centre sud du Vietnam

Table 2. Statistics of aquaculture in the south-central region.

\begin{tabular}{lcccc}
\hline Année & $\begin{array}{c}\text { Production } \\
\text { totale } \\
\text { (tonnes) }\end{array}$ & $\begin{array}{c}\text { Production } \\
\text { aquacole } \\
\text { (tonnes) }\end{array}$ & $\begin{array}{c}\text { Pourcentage } \\
\text { de production } \\
\text { aquacole } \\
\text { (\%) }\end{array}$ & $\begin{array}{c}\text { Surface } \\
\text { aquatique } \\
\text { exploitée } \\
\text { (hectares) }\end{array}$ \\
\hline 1995 & 339377 & 7873 & 2,31 & 14,5 \\
1996 & 349668 & 8911 & 2,54 & 14,0 \\
1997 & 381232 & 9674 & 2,53 & 14,7 \\
1998 & 384548 & 13290 & 3,46 & 18,8 \\
1999 & 441104 & 12832 & 2,90 & 20,3 \\
2000 & 462938 & 20031 & 4,32 & 19,0 \\
2001 & 484152 & 26054 & 5,38 & 22,1 \\
2002 & 520972 & 27561 & 5,29 & 23,9 \\
2003 & 546709 & 31493 & 5,76 & 26,4 \\
2004 & 582785 & 35811 & 6,14 & 27,1 \\
2005 & 623845 & 48914 & 7,84 & 25,2 \\
2006 & 628242 & 50009 & 7,96 & 27,2 \\
\hline
\end{tabular}

Source : statistiques extraites de l'annuaire statistique par province.

d'une validation préalable par des représentants du ministère qui leur ont reconnu un caractère exemplaire.

Une difficulté majeure a été très vite identifiée : il s'agit de l'insuffisance et de la mauvaise qualité des données statistiques officielles détaillées fournies par l'Office général statistique du Vietnam et l'office statistique du ministère vietnamien des Pêches. Pour tenter de pallier cette carence, nous avons complété les sources disponibles par des investigations originales, en réalisant des observations directes auprès des acteurs de l'approvisionnement.
Ces informations ont été recueillies, soit par des entretiens, soit à l'aide d'un questionnaire permettant de caractériser la relation verticale entre les intermédiaires et les entreprises dans l'approvisionnement de la matière première. Par ailleurs, des entretiens faits auprès des autres acteurs (pêcheurs, aquaculteurs, intermédiaires), à travers trois questionnaires en complément, ont eu pour objectif de mieux circonscrire le phénomène et ont permis de formuler des propositions solides. Ces informations ont été collectées avec soin, pour pouvoir en extraire des conclusions cohérentes et offrir un reflet fidèle de la situation.

\section{Résultats et discussions :}

Sur les quais, les intermédiaires procèdent aux opérations d'achat et de classification des prises, fournissent le marché local et les grandes entreprises. Sur chaque quai, on trouve de 7 à 12 intermédiaires (tableau 3).

Nous pouvons les classer en trois catégories : les détaillants, les semi-grossistes et les grossistes. Des grossistes fournissent en priorité l'exportation directe ou de grandes entreprises des autres régions.

Les résultats extraits des enquêtes ont démontré que les intermédiaires de la région jouent un rôle important dans l'approvisionnement, sans pour autant être spécialisés dans ce domaine. Le niveau d'étude moyen de ces acteurs est le premier cycle secondaire. Ils ont créé des relations étroites avec les producteurs de produits aquatiques à travers les avances des prestataires (100\% des intermédiaires interrogés ont fait des avances en liquide aux pêcheurs et $45 \%$ parmi eux ont fait des avances en nature). Pas plus que les pêcheurs, ils ne sont pas encore bien équipés pour la conservation de la matière première. Les intermédiaires disposent rarement de camions réfrigérés. Nos enquêtes ont permis de mettre en exergue les relations particulières existant 
Tableau 3. Caractéristiques socio-économiques des acteurs de la filière de commercialisation des produits de la pêche.

Table 3. Socio-economic characteristics of middlemen.

\begin{tabular}{|c|c|c|c|c|c|c|c|c|c|}
\hline \multirow[t]{3}{*}{ Catégories } & & \multicolumn{8}{|c|}{ Fournisseurs } \\
\hline & & \multicolumn{2}{|c|}{ Intermédiaires } & \multicolumn{2}{|c|}{ Agents-achat } & \multicolumn{2}{|c|}{ Aquaculteurs } & \multicolumn{2}{|c|}{ Pêcheurs } \\
\hline & & 30 pers. & $\%$ & 13 pers. & $\%$ & 20 pers. & $\%$ & 20 pers. & $\%$ \\
\hline \multirow[t]{2}{*}{ Capital propre } & 100 (million VND) & 30 & 100 & 13 & 100 & - & - & - & - \\
\hline & $<100$ (million VND) & - & - & - & - & 20 & 100 & 20 & 100 \\
\hline \multirow[t]{5}{*}{ Niveau d'étude } & Professionnel & 1 & 3,3 & 7 & 53 & 5 & 25 & - & - \\
\hline & Lycée & 7 & 23,4 & 6 & 47 & 6 & 30 & 1 & 5 \\
\hline & Collège & 3 & 10,0 & - & - & 4 & 20 & 4 & 20 \\
\hline & Primaire & 10 & 33,3 & - & - & 2 & 10 & 6 & 30 \\
\hline & Aucun & 9 & 30,0 & - & - & 3 & 15 & 9 & 45 \\
\hline \multirow[t]{2}{*}{ Nombre de salariés } & 10 personnes & 22 & 73 & - & - & - & - & - & - \\
\hline & $<10$ personnes & 8 & 27 & 13 & 100 & 20 & 100 & 20 & 100 \\
\hline \multirow[t]{2}{*}{ Avance de prestataire } & En liquide & 30 & 100 & - & - & - & - & - & - \\
\hline & En nature & 5 & 45 & - & - & - & - & - & - \\
\hline \multirow[t]{2}{*}{ Mode de paiement } & En espèce & 30 & 100 & 13 & 100 & - & - & - & - \\
\hline & Autres formes & 8 & 27 & - & - & - & - & - & - \\
\hline \multirow[t]{3}{*}{ Vente à destination } & Entreprises & 30 & 100 & 13 & 100 & 8 & 40 & 4 & 20 \\
\hline & Intermédiaires & - & - & - & - & 12 & 60 & 16 & 80 \\
\hline & exportation & - & - & - & - & - & - & - & - \\
\hline \multicolumn{2}{|l|}{ Moyen de conservation } & \multicolumn{2}{|c|}{$\begin{array}{l}\text { Tonneau } \\
\text { de } 200 \text { litres }\end{array}$} & \multicolumn{2}{|c|}{$\begin{array}{l}\text { Tonneau } \\
\text { de } 200 \text { litres }\end{array}$} & \multicolumn{2}{|c|}{$\begin{array}{l}\text { Panier } \\
\text { en bambou }\end{array}$} & \multicolumn{2}{|c|}{$\begin{array}{l}\text { Navire équipé } \\
\text { d'un vivier }\end{array}$} \\
\hline \multicolumn{2}{|l|}{ Forme de conservation } & \multicolumn{2}{|c|}{$\begin{array}{l}\text { Conserver dans la } \\
\text { glace en paillette }\end{array}$} & \multicolumn{2}{|c|}{$\begin{array}{l}\text { Conserver dans la } \\
\text { glace en paillette }\end{array}$} & \multicolumn{2}{|c|}{$\begin{array}{l}\text { Conserver dans la } \\
\text { glace en paillette }\end{array}$} & \multicolumn{2}{|c|}{$\begin{array}{l}\text { Conserver dans la } \\
\text { glace en paillette }\end{array}$} \\
\hline \multicolumn{2}{|c|}{ Difficultés en conservation } & \multicolumn{2}{|c|}{$\begin{array}{l}\text { Qualité de la } \\
\text { matière première }\end{array}$} & \multicolumn{2}{|c|}{$\begin{array}{l}\text { Qualité de la } \\
\text { matière première }\end{array}$} & \multicolumn{2}{|c|}{$\begin{array}{l}\text { Caractéristiques } \\
\text { des produits } \\
\text { aquatiques }\end{array}$} & \multicolumn{2}{|c|}{$\begin{array}{l}\text { Manque de capital } \\
\text { pour équiper les } \\
\text { réfrigérateurs }\end{array}$} \\
\hline
\end{tabular}

Source : données extraites des questionnaires auprès des acteurs dans l'approvisionnement en produits aquatiques des entreprises sur le terrain.

entre les trois types d'acteurs : pêcheurs; intermédiaires ; entreprises.

Le schéma de la figure 1 pourrait représenter ces relations.

\section{Relations entre pêcheurs et intermédiaires}

Les intermédiaires prêtent de l'argent aux pêcheurs pour qu'ils puissent construire leurs navires, les motoriser et les équiper en engins de pêche. Le montant du prêt dépend soit de la taille du bâtiment, soit de la capacité professionnelle ou du prestige du pêcheur. Selon nos enquêtes, dans la province de Phu Yen, les intermédiaires peuvent s'approprier, avec seulement 40 à 60 millions de dôngs vietnamiens (VND) - soit l'équivalent de 2000 à 3000 euros -, presque toutes les prises d'un bateau de pêche. De plus, ce prêt ne nécessite aucune formalité juridique : il n'est besoin que d'un document écrit avec la signature du propriétaire du navire et du prêteur. Le prêt sans taux d'intérêt offre à l'intermédiaire la possibilité d'acheter les prises et de bénéficier d'une commission. Le pêcheur a alors l'obligation de vendre aux intermédiaires ses prises au prix du marché

Il n'est cependant ni obligatoire ni mécanique que l'intermédiaire perçoive une commission, commission dont le taux peut d'ailleurs varier selon les circonstances. Ainsi, si le pêcheur ne peut ponctuellement pas faire face à ses frais, cette commission est supprimée. En revanche, si les prises sont en grande quantité et de grande valeur, l'intermédiaire prélève une commission d'un montant plus élevé. La souplesse en matière de fixation de la commission varie en fonction de la négociation entreprise dès la vente du poisson.

En contrepartie des bénéfices réalisés sur les prises du bateau ayant fait l'objet de l'investissement, l'intermédiaire a la responsabilité d'assurer la vente des produits, même pendant la saison de surpêche. Dans ce dernier cas, l'intermédiaire doit convaincre les entreprises de les acheter. Dans l'hypothèse où la négociation n'aboutirait pas, le pêcheur a le droit de vendre ses produits à un autre intermédiaire en remboursant la commission au premier (figure 2).

L'intermédiaire est également responsable du suivi du paiement par les entreprises, afin de rémunérer les pêcheurs. Comme les entreprises de transformation achètent fréquemment à crédit, il se trouve alors dans l'obligation d'avancer des fonds aux pêcheurs. C'est évidemment nécessaire lorsque les pêcheurs ont besoin d'argent pour rémunérer les hommes d'équipage, réparer le navire et préparer une nouvelle campagne de pêche. Dans le cas où le bâtiment doit être mis à terre pour une réparation lon- 


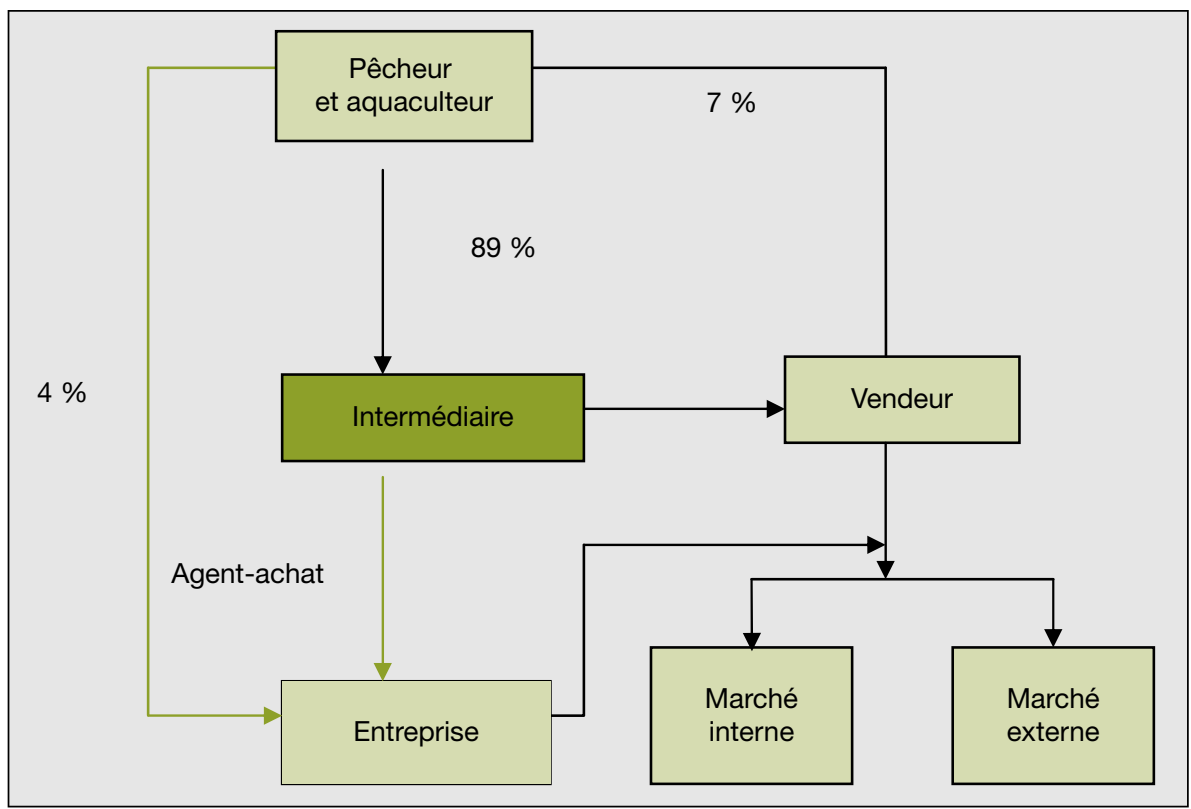

Figure 1. Filière d'approvisionnement en produits aquatiques de la région du Centre sud du Vietnam.

Figure 1. Supply system of fish product in the central-south region.

Ces données proviennent d'un échantillon stratifié de nos enquêtes sur le terrain.

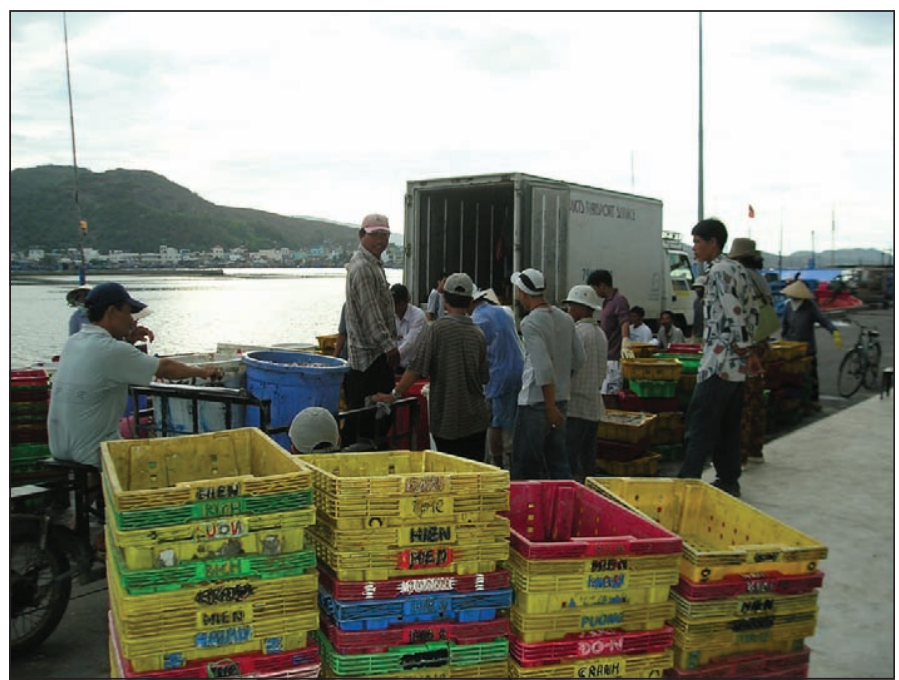

Figure 2. L'activité d'achat des intermédiaires sur un quai de la région du Centre sud du Vietnam

Figure 2. Buying activities of middlemen on wharfs in the central-south region.

gue et coûteuse, l'intermédiaire pourra éventuellement prêter des fonds au pêcheur afin qu'il s'acquitte de ses frais.

Ainsi, l'attachement indissoluble entre les intermédiaires et les pêcheurs est établi en raison de la proximité communautaire. Le "prêt-avance " rend le prix d'achat négocié par des intermédiaires plus concurrentiel que celui des entreprises de transformation.

\section{Relations entre l'intermédiaire et l'entreprise}

Les poissons sont vendus à des intermédiaires qui ont déjà financé les pêcheurs pour équiper leurs navires de pêche, pour acheter l'huile, l'essence ou l'eau potable. On pourrait envisager que des propriétaires de navires puissent ne pas accepter cet investissement qui leur semble bien modeste et, par conséquent, vendent directement leurs produits aux entreprises. Mais ce type de vente, pour être réalisé, se heurterait à bien des démarches compliquées et difficiles à résoudre. Par ailleurs, beaucoup d'entreprises souhaitant entretenir des bonnes relations avec les intermédiaires se refusent à avoir des contacts avec les pêcheurs, ce qui rend encore plus difficile la situation de ces derniers (tableau 4).

Après avoir acheté au seau (unité de mesure couramment utilisée) les poissons débarqués, les intermédiaires les classent en plusieurs catégories selon la demande des entreprises. Les poissons qui répondent aux normes de matières premières pour la transformation halieutique sont transportés vers l'entreprise. Le reste est vendu aux commerçants du marché local ou à des acheteurs de poissonrebut. Dans certains cas, le poisson pourra, en fonction des contrats conclus par les pêcheurs et les intermédiaires, être vendu directement aux détaillants ou à des acheteurs sur le marché local. Dans ce cas de figure, les intermédiaires perçoivent des détaillants ou des ramasseurs une commission perçue sur chaque kilogramme de poisson vendu. Le montant habituel est de 0,025 à 0,03 euro pour les petits maquereaux ou les sardines; de 0,01 à 0,02 euro pour le poissonrebut.

L'entreprise, pour sa part, choisit un ou plusieurs intermédiaires qui opéreront sur les différents quais en qualité d'agents d'achat. Il n'y a pas de contrat entre eux ; seuls un engagement et la confiance mutuelle fondent leur collaboration.

L'entreprise doit informer les intermédiaires sur le prix des matières premières, en fonction de la taille, des normes de qualité et de la quantité d'achat. Si un changement dans la fixation du prix d'achat intervient, l'information doit être rapidement transmise à l'intermédiaire.

Lorsque le prix d'achat fixé par l'entreprise est plus bas que celui de ses concurrents, l'intermédiaire doit évidemment négocier avec elle. Si la quantité des prises est très importante, l'entreprise doit néanmoins assumer leur achat, quitte à augmenter la puissance des machines, le temps de travail et le volume des entrepôts nécessaires à leur conservation.

Pour sa part, l'intermédiaire a l'obligation de fournir des matières premières correspondant aux critères de l'opération de transformation des entreprises partenaires. C'est le facteur principal de sa notoriété. En 
Tableau 4. L'approvisionnement en matière première des fournisseurs de l'entreprise.

Table 4. Supply of raw material from tradesmen to enterprise.

\begin{tabular}{|c|c|c|c|c|c|c|}
\hline & \multicolumn{2}{|c|}{ En 2003} & \multicolumn{2}{|l|}{ En 2004} & \multicolumn{2}{|l|}{ En 2005} \\
\hline & $\begin{array}{c}\text { Quantité } \\
\text { (kg) }\end{array}$ & $\%$ & $\begin{array}{l}\text { Quantité } \\
\text { (kg) }\end{array}$ & $\%$ & $\begin{array}{c}\text { Quantité } \\
\text { (kg) }\end{array}$ & $\%$ \\
\hline $\begin{array}{l}\text { 1. Intermédiaires } \\
\text { (Middlemen) }\end{array}$ & 1763255 & 81,23 & 2143920 & 82,07 & 2600398 & 81,12 \\
\hline $\begin{array}{l}\text { 2. Pêcheurs } \\
\text { (Fishmen) }\end{array}$ & 11070 & 0,51 & 4506 & 0,17 & 20515 & 0,64 \\
\hline $\begin{array}{l}\text { 3. Agents-achat } \\
\text { (Tradesmen) }\end{array}$ & 354908 & 16,35 & 438345 & 16,78 & 520272 & 16,23 \\
\hline $\begin{array}{l}\text { 4. Aquaculteurs } \\
\text { (Fish-farmers) }\end{array}$ & 41460 & 1,91 & 25535 & 0,98 & 64432 & 2,01 \\
\hline
\end{tabular}

Source : statistiques sur les matières premières de Long Sinh - une entreprise typique de transformation halieutique de taille moyenne dans la région du Centre sud du Vietnam (statistiques internes de l'entreprise collectées sur place par enquête)

fonction de celle-ci, leur collaboration prendra la forme suivante : l'entreprise a pour priorité le paiement du prix et l'investissement dans les équipements des intermédiaires partenaires. En contrepartie, l'intermédiaire collecte les prises des bateaux de pêche et les fournit régulièrement à l'entreprise partenaire, même en cas de retard de paiement.

\section{Billan et suggestions}

On peut donc conclure que les intermédiaires jouent un rôle essentiel dans le système d'approvisionnement en matières premières des entreprises de transformation halieutique de la région du Centre sud du Vietnam, tout comme dans les provinces plus au Nord (Mc Cullough et Phung Giang, 2001) ou encore dans la province chinoise de Hubey (Wang, 1999).

Ils sont partie intégrante de la chaîne d'approvisionnement de l'entreprise, et occupent même une place primordiale dans le fonctionnement de celle-ci. En effet, grâce à eux, les pêcheurs peuvent avoir accès au capital, ce qui les aide à développer leur activité. C'est d'autant plus nécessaire que les banques ne veulent pas investir dans ce domaine, considéré comme à risques en raison des pertes subies.

Leur fonctionnement n'est pas alourdi par les démarches administratives et les négociations. Il est efficace en matière de paiement et contribue à réduire le temps de transport des produits jusqu'à l'entre- prise, assurant ainsi la qualité des matières premières.

Leur aspect négatif tient à l'influence de leur activité " achat-vente " dans le marché des poissons des entreprises. Avec un modeste investissement, un intermédiaire peut bénéficier d'une commission qui augmente considérablement à condition que les prises soient abondantes, et ceci jusqu'à ce que le pêcheur puisse lui rendre la somme d'argent empruntée. Par conséquent, ils s'enrichissent rapidement, alors que les pêcheurs sont confrontés à de plus en plus de difficultés, d'autant que ceux qui ne peuvent avoir des relations étroites avec l'entreprise sont obligés de vendre à bas prix aux intermédiaires.

Si cette activité peut se révéler fructueuse, elle n'en présente pas moins des risques : l'intermédiaire risque de perdre tout l'argent qu'il a investi si le pêcheur s'enfuit avec son navire, vend à un autre intermédiaire ou, en cas de perte financière, se fait saisir par les banques. Cependant il convient de relativiser cet aléa: la somme investie est modique et souvent trop faible pour améliorer la capacité de prise des pêcheurs.

Les intermédiaires, bien qu'ils n'aient aucune influence sur le volume des prises, ont cependant le droit de fixer les prix. Cela rend instable le prix des poissons et influe considérablement sur l'efficacité des pêcheurs, et par conséquent sur l'activité des entreprises. Aussi, malgré la production croissante de produits aquatiques dans la région, les entreprises ne disposent pas d'assez de poissons pour fonctionner correctement (Le Anh T, Hambrey J, 2005).
Par ailleurs, en raison de l'intervention de l'intermédiaire, l'entreprise ne peut pas gérer correctement la qualité des matières premières qui lui sont fournies. Le suivi et la traçabilité des produits finis depuis l'origine sont une question qui devrait se poser dans les entreprises de transformation halieutique du Vietnam.

Afin d'améliorer la situation, nous formulerons quelques propositions à court terme.

\section{S'inscrire dans une perspective de développement durable}

Pour un développement durable de la transformation des produits halieutiques, il faudrait une coordination et une synchronisation de plusieurs filières en s'appuyant sur une évaluation fiable des stocks pouvant être pêchés afin de les gérer de manière optimale. La réalisation de l'inventaire des quais de déchargement dans cette région est également importante, sinon primordiale.

Par ailleurs, en raison de la distance entre les quais de déchargement et les usines de la région, il conviendrait que les provinces financent l'établissement d'entrepôts ad boc près des quais pour préserver la qualité des produits pêchés.

À long terme, quelques marchés de gros devraient s'établir dans la région afin d'éliminer la situation actuelle de concurrence entre les intermédiaires.

Il semble aussi absolument nécessaire de réglementer l'activité des intermédiaires :

- en les obligeant à enregistrer leurs activités de commerce pour mieux contrôler leur fixation de leur prix et le paiement de leurs impôts ;

- en leur demandant d'améliorer l'équipement et les moyens de manutention, ainsi que la conservation des matières premières.

À l'intérieur du système d'approvisionnement, il convient que l'entreprise sélectionne les intermédiaires qui paraissent le mieux convenir aux objectifs marketing et aux intérêts du producteur et des pêcheurs. Bien que plusieurs méthodes soient utilisables pour effectuer les choix, l'analyse multicritère conserve le double avantage de la rigueur et de la souplesse. Il s'agit de : connaître le marché en "amont "; exprimer les besoins et provoquer l'offre ; évaluer, sélectionner les intermédiaires; négocier au mieux avec les intermédiaires; commander de 
manière fonctionnelle ; assurer le suivi et la réception de la commande.

Dans la mise en place d'une logique intégrée, les flux sont appréhendés des fournisseurs aux consommateurs ; l'approvisionnement n'est alors qu'un maillon de la chaîne. Dans ce contexte, l'organisation efficace des flux de matière première de l'entreprise interdit tout cloisonnement (Bourbonnais et Vallin, 1995). Cette vue globale de l'écoulement du flux permet de travailler sur les approches de partenariat avec le fournisseur. Le partenariat avec les intermédiaires implique une planification glissante, une remise à jour permanente du plan et de l'évaluation des plages d'imprécision.

\section{Inciter à l'intégration verticale des intermédiaires}

Plusieurs recherches ont permis de conclure sur des figures mixtes de l'intégration verticale dans le secteur agricole. Ce courant est désigné par l'expression anglo-saxonne de Raising Rivals Costs (RRC) qui désigne l'élévation des coûts des concurrents (Salop et Scheffman, 1983, 1987; Krattenmaker et Salop, 1986, 1987).

L'intégration verticale est, en règle générale, jugée porteuse d'efficacité. Dans un état donné de technologie, elle est tout d'abord supposée conduire à un accroissement de l'offre et à une baisse de prix, alors même que les firmes en amont sont en position de monopole sur leurs marchés.

Le secteur de la conserve de poissons en France a ainsi été l'objet d'un affrontement juridico-économique pour la prise de contrôle du principal fournisseur du secteur (société Saupiquet versus société Sea Deal). Or, la prise de contrôle de ce fournisseur a entraîné des contraintes d'approvisionnement pour les autres entreprises du secteur.

La stratégie d'intégration verticale n'est toutefois pas sans effet sur la situation des firmes non intégrées. On peut ainsi relever au moins six types de stratégies susceptibles de les mettre en difficulté (Glais, 1995).

Le cas de l'industrie du thon examiné ici par Guillotreau constitue un exemple tout à fait illustratif du risque de forclusion dont peuvent être victimes les entreprises non intégrées, et ce plus particulièrement lorsque l'intégration met en jeu des " infrastructures essentielles ", c'est à dire de ressources productives :
- dont les entreprises situées en aval ne peuvent se passer ;

- qu'elles sont incapables de dupliquer ; - dont l'offre est contrôlée par un ou quelques opérateurs dominants (Glais, 1999).

Dans le cadre de cet article, nous voulons proposer un mécanisme d'intégration verticale s'appliquant au modèle de coopératives, le modèle le plus adapté, semble-t-il, à notre recherche d'une amélioration de la contribution des intermédiaires dans l'approvisionnement en produits aquatiques des industries de transformation halieutique.

La coopérative permet d'améliorer la synergie des acteurs de l'approvisionnement en matière première des entreprises de transformation halieutique des PED. Si les producteurs, les fournisseurs de matière première et l'entreprise s'organisent en coopérative, ils auront l'obligation de collaborer. La stabilité des échanges entre l'entreprise, l'intermédiaire et les pêcheurs sera assurée par la détermination d'un prix conventionnel, faisant ainsi disparaître le caractère superficiel et incertain de leur relation.

Dans la mesure où les coopératives ont une activité d'approvisionnement nettement supérieure à celle des entreprises privées, il existe une faiblesse relative de la valeur ajoutée dégagée sur les achats par rapport aux performances des entreprises de droit commun.

Le modèle de coopérative adapté aux conditions de production du Vietnam pourrait être fondé sur la définition générale de l'Alliance coopérative internationale : "Une coopérative est une association autonome de personnes volontairement réunies pour satisfaire leurs aspirations et besoins économiques, sociaux et culturels communs au moyen d'une entreprise dont la propriété est collective et où le pouvoir est exercé démocratiquement" (Bidet, 1998).

Il nous faut donc concevoir la coopérative comme un statut rassemblant les activités de plusieurs individus dans des secteurs différents (commerce, consommation des produits, services). Autrement dit, les activités économiques, culturelles, sociales des individus constituent la base de la coopérative. La coopérative ne répond pas à tous les besoins de ses coopérateurs mais seulement à ceux qui leur sont communs et qui seront mieux réalisés grâce à la coopération. La coopérative et ses adhérents forment donc un marché dans lequel ils sont clients les uns les autres.
À partir des analyses empiriques, nous réfléchirons à un modèle de coopérative plus adapté au secteur de la pêche, plus dynamique et plus ouvert. Le mécanisme assurant la pérennité de la coopérative sera l'actionnariat. La coopérative "anonyme " n'est pas un modèle décrit par la typologie classique. Assez proche de la société anonyme classique, cette forme de coopérative a été présentée au ministère des Pêches par l'Institut de la planification des pêches en 2008.

Le modèle proposé, celui de la " coopérative anonyme " relève alors de l'économie de coopération dans la chaîne de valeur. Le concept de chaîne de valeur a été introduit par Porter dans son ouvrage L'avantage concurrentiel (Porter, 1986). Lorsqu'une entreprise n'est pas parfaitement intégrée verticalement, sa chaîne de valeur s'insère dans une chaîne plus globale reliant les chaînes des fournisseurs et des circuits de distribution jusqu'au client. L'avantage concurrentiel peut être augmenté par une meilleure coordination de l'ensemble de ses chaînes qui vont de l'origine du produit ou service jusqu'au client.

Mais, compte tenu de son caractère novateur, la création d'un tel type de coopérative ne peut s'envisager que sur le long terme.

En même temps que le développement de l'intégration verticale, les intermédiaires seront restreints en nombre de statuts mais deviendront spécialisés dans leurs activités.

\section{Conclusion}

Au terme de cette étude sur l'influence des activités commerciales des intermédiaires dans le marché des matières premières de l'industrie de transformation de la région, une évidence s'impose : en raison de leur faible assise financière, les intermédiaires n'ont d'autre choix que de définir une politique de gestion optimale.

En revanche, le nombre limité de grossistes, leurs actions concertées en matière d'offre de prix qui relèvent de l'entente illicite, ainsi que leur solidarité, participent à la négation des principes fondant la structure de la compétitivité du marché. On notera enfin qu'avec la croissance de l'élevage commercial en étang, un nouveau modèle est en voie d'apparition dans la chaîne de commercialisation 
(Alam, 2000). Il pourrait modifier sensiblement les notions de zones de production, de lieu de première mise sur le marché, de marchés intermédiaires, de zones de consommation et de marchés de détail.

\section{Références}

Alam S. Production, accessibility and consumption patterns of aquaculture products in Bangladesh. Report submitted to ICLARM. Penang (Malaysia) : ICLARM, 2000.

Bidet E. L'économie sociale: un secteur d'avenir ? Problèmes politiques et sociaux 1998; $798: 1-77$.

Dey MM. Analysis of demand for fish in Bangladesh. Aquaculture Economics and Management 2000a ; $4: 63-82$.
Dey MM, Rab MA, Paraguas FJ, et al. Fish suply and demand in Asia : Progress report. Penang (Malaisie) : World Fish center, 2002.

Glais M. Les relations discriminatoires dans les relations fournisseurs-distributeurs. Economie Industrielle $1995 ; 72: 81-97$.

Glais M. Préface. Les Cahiers de l'Artemis $1999 ; 1: 1-10$

Krattenmaker TG, Salop SC. Analyzing anticompetitive exclusion. Antitrust Law J 1987 ; 56 : 71-89.

Krattenmaker TG, Salop SC. Competition and Cooperation in the Market for Exclusionary Rights. Am Econ Rev 1986 ; 76 : 109-13.

Mc.Cullough B, Phung Giang $H$. The live reef fish trade in Vietnam: a preliminary report from the field. SPC live reef fish information Bulletin $2001: 8 ; 12-6$

Mafimisebi TE, Okunmadewa FY. Are middlemen really exploitative? Empirical evidence from the sun dried fish market in Southwest, Nigeria. Electronic Proceedings of the 13th Biennal international Conference of the IIFET 2006, Portsmouth (UK), 11-14 Juillet 2006.
Guillotreau P, Le Roy F. Les comportements concurrentiels comme objet d'étude (Concurrence et intégration verticale: une approche institutionnelle). Les cahiers de l'Artemis $1999 ; 1: 46-66$

Porter M. L'avantage concurrentiel. Paris : InterEditions, 1986.

Bourbonnais R, Vallin P. Comment optimise les approvisionnements. Paris: Economica, 1995.

Salop SC, Scheffman DT. Cost-Raising Strategies. J Ind Econ 1987 ; 16 : 19-34.

Salop S, Scheffman D. Raising Rivals' Costs. Am Econ Rev 1983; 73 : 267-71.

Thai Thanh D. Thuy san Viet Nam - Nhung chang duong phat trien. Document en ligne du Ministère des Pêches.2005. http://www.ficen.org.vn/details. asp?object=48\&news_ID=112270338)

Wang N. Transaction Costs and the Structure of the Market: A Case Study. Am J Econ Social $1999 ; 58: 783-806$ 